Typ-2-Diabetes

\title{
Patienten profitieren von frühzeitigem Einsatz von GLP-1-Analoga
}

Inkretinmimetika wie Liraglutid (Victoza ${ }^{\circledR}$ ) können die Blutzuckerkontrolle bei oral unzureichend eingestellten Patienten mit Typ-2-Diabetes deutlich verbessern - bei geringem Risiko für Hypoglykämien und potenzieller Gewichtsreduktion. Neue Studiendaten belegen, dass Patienten mit Typ2-Diabetes von einem frühzeitigen Einsatz des GLP (Glucagon-like Peptide)-1-Analogons besonders profitieren und die Therapiezufriedenheit der Patienten trotz Beginn einer Injektionstherapie steigt.

„Eine Kombination von Liraglutid mit oralen Antidiabetika (OAD) ist sowohl im frühen als auch im fortgeschritteneren Stadium eines Typ-2-Diabetes wirksam“, fasste Prof. David Matthews aus Oxford die gepoolten Daten von 7 randomisierten klinischen Studien mit insgesamt 4.625 Patienten mit Typ-2-Diabetes zusammen [1]. Bei Einsatz im frühen Stadium besteht aber das Potenzial, Blutzuckereinstellung ( $\mathrm{HbA}_{1 \mathrm{c}}$-Wert) und Betazellfunktion besonders deutlich verbessern zu können.

Die Studienteilnehmer wurden für die neue Meta-Analyse der Liraglutid-Studiendaten nach ihrer bisherigen Therapie in 2 Gruppen eingeteilt: Patienten, die bisher nur mit Lebensstiländerungen oder maximal einem OAD behandelt wurden, galten als Personen im frühen Diabetesstadium; im Mittel hatten sie seit 6 Jahren Diabetes; Patienten, die bereits mit mindestens 2 OADs behandelt wurden, wur-

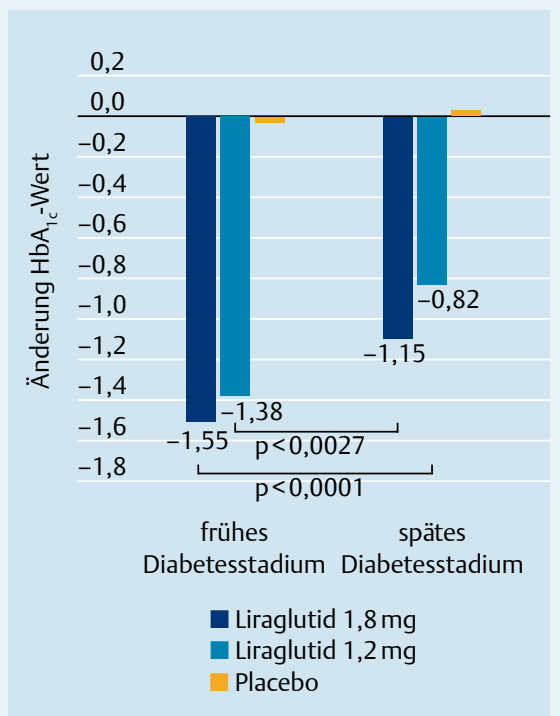

Abb. 1 Änderung des $\mathrm{HbA}_{1 c}$-Wertes bei Patienten, die im frühen bzw. späten Diabetesstadium behandelt werden. den der fortgeschritteneren Gruppe zugeteilt; die Diabetesdauer betrug bei ihnen im Mittel 9 Jahre. Der $\mathrm{HbA}_{1 \mathrm{c}}$-Wert lag bei allen Patienten bei rund 8,5\%. Über 26 Wochen wurden die Studienteilnehmer zusätzlich mit Liraglutid $1,2 \mathrm{mg}$ oder $1,8 \mathrm{mg}$ täglich oder Placebo behandelt (zu beachten: zugelassen ist das GLP-1-Analogon nur in Kombination mit Metformin bzw. einem Sulfonylharnstoff).

Der $\mathrm{HbA}_{1 \mathrm{c}}$-Wert nahm bei Patienten im frühen Diabetesstadium dosisabhängig um 1,55 bzw. 1,38\% ab, im späteren Stadium um 1,18 bzw. 0,82\% (Placebo -0,46 bzw. $-0,09 \%$ ). $72 \%$ der frühzeitig mit Liraglutid $1,8 \mathrm{mg}$ täglich behandelten Patienten erreichten den $\mathrm{HbA}_{1 c}$-Zielwert $<7 \%$, in der fortgeschritteneren Gruppe nur 49\% ( $p<0,0001$ ). Bei den mit Liraglutid 1,2 mg täglich behandelten Patienten war der Unterschied von 61 zu $41 \%$ statistisch nicht signifikant (Abb. 1).

Außerdem erholte sich auch die BetazellFunktion bei frühzeitigem Einsatz von Liraglutid besser, berichtete Matthews. Die Werte beim HOMA-B-Test verbesserten sich unter der höher dosierten Liraglutid-Therapie um $41 \%$ bei früh im Vergleich $\mathrm{zu} 24 \%$ bei spät behandelten Patienten ( $p=0,0037)$.

\section{Therapiezufriedenheit der Behandelten} steigt

Der Wechsel von einer rein oralen Diabetestherapie auf eine zusätzliche Injektionstherapie hat keinen ungünstigen Einfluss auf die Therapiezufriedenheit der Patienten. Dies ist in einer weiteren Studie mit Liraglutid belegt worden, deren Ergebnisse ebenfalls aktuell beim europäischen Diabeteskongress in Lissabon vorgestellt worden sind [2]. Ganz im Gegenteil: Die in der Studie vollzogene Therapieumstellung vom oralen DPP-IVInhibitor Sitagliptin auf das s.c. zu applizierende GLP-1-Analogon, beides kombiniert mit Metformin, war mit einer Verbesserung der Gesamtzufriedenheit verbunden, berichtete Prof. Eduard Montanya, Barcelona. Dieser Effekt könnte nach seiner Ansicht auf die bessere Blutzuckerkontrolle und die stärkere Gewichtsreduktion unter Liraglutid $(1,2 \mathrm{mg}$ oder 1,8 mg täglich) zurückzuführen sein, die bereits in einem offenen Vergleich mit Sitagliptin (100 mg täglich) bei 665 Pa- tienten mit Typ-2-Diabetes über 52 Wochen belegt worden sind [3]. Bei Studienende hatte sich der $\mathrm{HbA}_{1 \mathrm{c}}$ unter Liraglutid $1,2 \mathrm{mg}$ täglich um 1,3\% reduziert im Vergleich zu nur 0,9\% unter Sitagliptin. Das Körpergewicht hatte sich um $2,8 \mathrm{~kg}$ verringert vs. $1,2 \mathrm{~kg}$ in der Vergleichsgruppe. Die neuen Daten zur Therapiezufriedenheit basieren auf einer weiteren Extensionsphase dieser Untersuchung über 26 Wochen bei insgesamt 337 Patienten, die von Sitagliptin auf Liraglutid 1,2 mg oder $1,8 \mathrm{mg}$ täglich umgestellt worden sind. Nach der Umstellung verringerte sich das Körpergewicht der Behandelten um weitere $1-1,5 \mathrm{~kg}$, berichtete Montanya, und der Anteil der Patienten, die den $\mathrm{HbA}_{1 \mathrm{c}}{ }^{-}$ Zielwert $<7 \%$ erreichten, nahm von 30 auf $50 \%$ zu. Die Therapiezufriedenheit der Patienten vor der Umstellung und weitere 26 Wochen nach der Umstellung wurde bei 337 Patienten per Fragebogen (Diabetes Treatment Satisfaction Questionnaire, 8 items, Score jeweils 0-6) erfragt. Mit der Flexibilität und Convenience der Therapie waren die Patienten unter Sitagliptin und Liraglutid gleichermaßen zufrieden. Die Gesamtzufriedenheit der Patienten, die auf Liraglutid $1,2 \mathrm{mg}$ täglich umgestellt worden waren, war signifikant gestiegen (Verbesserung des Gesamtscores von 31,8 auf 33,2 Punkte). Patienten unter Liraglutid 1,2 mg erzielten auch höhere Scorepunkte bei den Fragen nach der Zufriedenheit mit der Therapie sowie den Wahrscheinlichkeiten, die Therapie zu empfehlen oder fortzusetzen. Auch die subjektive Wahrnehmung von hyperglykämischen Episoden war nach Umstellung auf Liraglutid verringert. Keine signifikanten Unterschiede gab es in den Bewertungen zwischen den Patienten aus den Gruppen mit 1,2 mg oder 1,8 mg täglich.

Roland Fath, Frankfurt

\section{Literatur \\ 1 Poster 791, EASD 2011 \\ 2 Poster 796, EASD 2011 \\ 3 Pratley R et al. Diabetes 2011}

Quelle: Symposium „Incretins in diabetes: from pathophysiology to therapeutic use", 12. September 2011, Novo Nordisk Media Update, 14. September 2011 im Rahmen der 47. Jahrestagung der European Association for the Study of Diabetes (EASD) in Lissabon. Der Text entstand mit freundlicher Unterstützung durch Novo Nordisk 


\section{Diabetes Management Report Deutschland 2010}

Der Diabetes Management Report $2010^{1}$ zeigt im Vergleich zu einer ähnlichen Befragung aus dem Jahr $2006^{2}$ deutliche Fortschritte auf dem Gebiet des eigenverantwortlichen Selbstmanagements bei Diabetes. „Mittlerweile führt mit $81 \%$ $(n=813)$ eine große Mehrheit der 1004 befragten Menschen mit Diabetes regelmäßige Blutzuckermessungen durch“, sagt Prof. Theodor Koschinsky, Diabetologe an der Heinrich-Heine-Universität Düsseldorf, zu der Befragung. „Außerdem zeigt die Umfrage, dass die Befragten sich zunehmend an strukturierte Messschemata halten, die Werte notieren und Handlungen daraus ableiten - sowohl alleine als auch mit Unterstützung der Ärzte.“

\section{Sinnvoll messen mit individuellen} BZSK-Konzepten

Positiv ist zu bewerten, dass sich Menschen mit Diabetes mehr als noch 2006 für individuelle BZSK-Konzepte entscheiden. Sie messen beispielsweise nicht mehr bevorzugt nach einem starren Messschema morgens ihren Nüchternblutzucker (44\% von 813 Befragten im Jahr 2010 im Vergleich zu 80\% des Kollektivs von 966 Patienten im Jahr 2006). „Heutzutage messen Menschen mit Diabetes häufiger strukturiert, um aussagekräftige Tagesprofile zu erstellen. Dabei orientieren sie sich an stoffwechselrelevanten Ereignissen wie Mahlzeiten oder körperlicher Bewegung, anstatt allein ihrem subjektiven Gefühl zu folgen“, berichtet Koschinsky mit Blick auf die aktuelle Erhebung. Die von der Firma Roche Diagnostics Deutschland unterstützte telefonische Befragung zeigt gleichzeitig, dass Menschen mit Diabetes keineswegs unnötige oder überflüssige Messungen durchführen. Vielmehr messen die Befragten angepasst an ihre jeweilige Therapieform unterschiedlich häufig ihren Blutzucker: ICT-Anwender messen mit

\footnotetext{
1 Koschinsky T. Diabetes Management Report Deutschland 2010 - Entwicklungen und Handlungsbedarf. Diab Stoffw und Herz 2011; 20: 135-142

2 Im Vergleich zu einer ähnlichen Befragung aus dem Jahre 2006: Koschinsky T: Blutzuckerselbstmanagement-Report Deutschland 2006 offenbart Wissens- und Handlungsdefizite. Diab Stoffw und Herz 2007; 16: 185-192
}

86\% (Basis $\mathrm{n}=141$ ) am häufigsten, Diätpatienten mit 3\% (Basis $n=120$ ) am seltensten mehrmals täglich ihren Blutzucker.

Arzt und Diabetesberater haben großen Einfluss auf Dokumentation

Auch beim Umgang mit ihren Diabetesdaten ist die Mehrheit der messenden Befragten gewissenhaft: $63 \%$ (Basis $n=813$ ) dokumentieren ihre Messwerte fast immer, überwiegend in handschriftlicher und gelegentlich auch in elektronischer Form. Auf das Dokumentationsverhalten hat der Behandler großen Einfluss, denn 59\% (Basis $n=592$ ) der Umfrageteilnehmer, die zumindest gelegentlich ihre Messergebnisse dokumentierten, notieren diese vor allem auf Wunsch ihres Arztes oder Diabetesberaters. Eine moderne, computergestützte Auswertung der Messergebnisse in Form von Grafiken wird allerdings erst von $10 \%$ (Basis $n=592$ ) der Befragten, die ihre Werte auch dokumentieren, genutzt. Koschinsky hofft, dass Menschen mit Diabetes und ihre Therapeuten das große Potenzial der grafischen Datenaufbereitung für die Therapieoptimierung künftig stärker ausschöpfen: „Die meisten Menschen haben eine ausgeprägte visuelle Wahrnehmung. Eine farbige Grafik mit Verlaufskurven ist viel leichter zu erfassen als eine Tabelle voll abstrakter Zahlen und kann wertvolle Impulse für das Beratungsgespräch zwischen Arzt und Patient liefern.“

\section{BZSK-Ergebnisse verstehen} und Lebensstil anpassen

Die Kommunikation zwischen Therapeuten und ihren Patienten hat sich in den vergangenen Jahren bereits deutlich verbessert: Während 2006 nur 43\% (Basis $\mathrm{n}=649$ ) der messenden und dokumentierenden Befragten mit BZSK angaben, dass ihr Arzt die Therapie auf Basis der gemeinsamen Analyse anpasst, berichten 2010 bereits $72 \%$ (Basis $n=592$ ) der BZSK-Anwender, dass ihr Therapeut auf Basis der Messwerte Verhaltensänderungen empfehle. Auch die eigenverantwortliche Übertragung ihrer BZSK-Ergebnisse in entsprechende Verhaltens- und Therapieänderungen gelingt immer mehr Menschen mit Diabetes: Unter den Befragten, die ihren Blutzucker kontrollieren und zumindest gelegentlich dokumentieren, reagieren mittlerweile $75 \%$ (Basis $n=592$ ) auf ihre Werte, indem sie ihren Lebensstil anpassen. 2006 hingegen wurden nur 52\% (Basis $n=649$ ) durch die Dokumentation zu einer gesünderen Lebensweise angespornt. Diese deutliche Steigerung zeigt, dass die intensive Aufklärung über die Vorteile des eigenverantwortlichen Diabetes Managements in den vergangenen Jahren erste Früchte trägt.

\section{Unterstützung für}

die Patientenberatung im Alltag

Im Fortbildungsprogramm Accu-Chek Forum lernen Ärzte und andere DiabetesFachleute, wie sie ein erfolgreiches Diabetes Management im Praxisalltag einsetzen und für die Therapie ihrer Patienten gezielt nutzen. Das Accu-Chek Forum 2011 soll Ärzten dabei helfen, die Therapie von Menschen mit Diabetes zu verbessern, ihre Patienten erfolgreich zu motivieren und das Praxismanagement zu erleichtern. Die Teilnehmer erhalten insbesondere auch Hilfestellung beim Einsatz elektronischer Dokumentationssysteme, welche die dokumentierten Messwerte übersichtlich grafisch aufbereiten und auf diese Weise das PatientenBeratungsgespräch erleichtern können. Auf Basis dieser Werte können sie dann gemeinsam mit ihrem Behandlungsteam - besser und schneller Handlungen zur Verbesserung ihrer Therapie ableiten.

Quelle : PM fischerAppelt, relations $\mathrm{GmbH}$, Frankfurt 


\section{SilverStar-Gewinner in Berlin ausgezeichnet}

Erstmals wurde in Berlin der mit insgesamt $25000 €$ dotierte Förderpreis „SilverStar" der Berlin-Chemie AG vergeben. Den 1. Preis und damit $15000 €$ erhielt das Theaterstück „Die Süße des Lebens“. Den 2. Preis teilte die Jury, die sich aus Vertretern der Bereiche Diabetologie und Altersmedizin zusammensetzt, auf 2 Projekte auf: das Diabetikersportprogramm „Difima - Diabetiker fit machen“ und „DiaKoPf - Diabetologische Kompetenz in Pflegeeinrichtungen“, sie erhielten je $5000 €$ Preisgeld.

Insgesamt reichten 43 Initiativen, Selbsthilfegruppen, Kliniken und Praxen ihre Projekte für den SilverStar-Förderpreis ein. Der Preis wurde in diesem Jahr erstmals ausgelobt und soll von nun an jährlich an Projekte und Initiativen vergeben werden, die älter werdenden Patienten mit Diabetes eine bessere Lebensqualität ermöglichen. Der SilverStar ist zudem ein wichtiger Bestandteil der neuen Initiative „Unsere Zukunft wird älter. Zeit zu handeln. “ der Berlin-Chemie AG, deren Gründung das Unternehmen im Rahmen der Preisverleihung bekanntgab.

Die Auszeichnung SilverStar der BerlinChemie AG soll vor allem diejenigen würdigen, die sich mit ihren Projekten, Versorgungskonzepten oder Services für ältere Diabetes-Patienten einsetzen. „Allen eingereichten Projekten gemeinsam ist der ganz persönliche Einsatz der Bewerber. Hier setzt der Förderpreis auch an: Er soll jene Bewerber und Projekte unterstützen, die sich die praktische Betreuung von älteren Menschen mit Diabetes zur Aufgabe gemacht haben. Die Resonanz auf die Ausschreibung war sehr groß. Das zeigt auch, wie wichtig eine solche Auszeichnung für die Bewerber ist“, betont der Jury-Vorsitzende Prof. Rüdiger Petzoldt, Bad Oeynhausen, im Rahmen der Preisverleihung.

\section{Die Süße des Lebens:}

Gelungene Mischung aus Unterhaltung und Information

Mit direkter Zielgruppenansprache und innovativem Ansatz, ein komplexes Krankheitsbild humorvoll und lebensbejahend zu vermitteln, überzeugte das Theaterstück „Die Süße des Lebens“. „Die Patienten und Angehörigen nehmen über die emotionale Ebene viele wertvolle
Informationen auf, können sich mit der anschließenden Expertenrunde austauschen und nehmen ein Stückchen mehr Lebensfreude mit nach Hause“, lobte Prof. Petzoldt. Das Theaterstück sei eine gelungene Mischung aus Unterhaltung und Information.

Die Cura Unternehmensgruppe, die das Stück beauftragt hatte, wollte vor allem ein informatives, qualitativ hochwertiges und zugleich unterhaltsames Angebot für ältere Menschen mit Diabetes entwickeln: „Wir haben uns die Frage gestellt, wie man außer mit Vorträgen, die wir auch regelmäßig in unseren Einrichtungen durchführen, Aufmerksamkeit für Diabetes wecken kann. Das Theaterstück sollte uns als Türöffner für Betroffene und deren Angehörige dienen“, erklärt Mareike Schüller von der Cura Unternehmensgruppe die Motivation für das Projekt. Umgesetzt wurde die Idee vom Galli Businesstheater. Rainer Eckhardt, Geschäftsführer der Galli Gruppe, schildert die positiven Zuschauerreaktionen: „Die Schauspieler berichten, dass die Aufführung für viele Besucher ein bewegendes Erlebnis ist und ihnen viele Anregungen gibt, auch im Alltag mehr die Lebensfreude und das Positive zu sehen."

Bewegung tut gut - das DIFIMA-Projekt „Difima - Diabetiker fit machen“ wurde von Detlev Kraft, selbst Sportstudiobetreiber, in Zusammenarbeit mit dem Sportstudionetzwerk INLINE und der Arbeitsgemeinschaft Diabetes und Sport der Deutschen Diabetes-Gesellschaft entwickelt. Difima ist ein 6-Wochen-Programm, das vor allem sportlich inaktive Typ-2-Diabetiker auf den „Geschmack am Sport“ bringen möchte. „Uns kam es darauf an, ein Projekt zu entwickeln, das Typ-2-Diabetikern die Schwellenangst vor einem Fitnessstudio nimmt.

Eine zeitlich begrenzte Aktion gezielt für Diabetiker senkt die Hemmschwelle, sich in ein Fitness-Studio zu begeben“, beschreibt Detlev Kraft das Projekt, das Erfolg hat: Die Teilnehmer stellen schnell fest, wie gut ihnen die Bewegung tut, und führen das Programm häufig auch nach Kursende weiter.

Insbesondere die altersgerechte Ansprache und das Eingehen auf die individuellen Gegebenheiten der Betroffenen
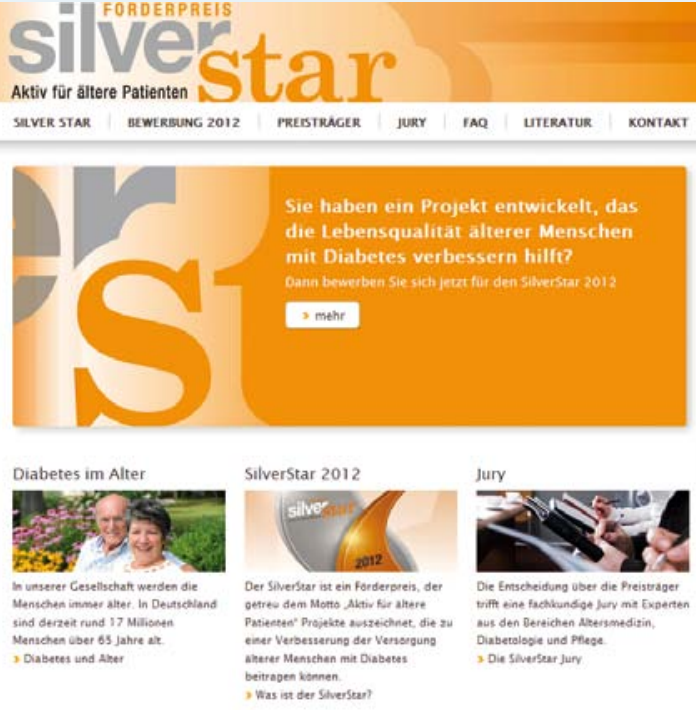

überzeugte die Jury von Difima. „Ein älter werdendes Klientel wird attraktiv an eine vermehrte Bewegung herangeführt. Difima sorgt im alltäglichen Leben für spürbare Verbesserungen. Mit den gesundheitsorientierten Fitnessstudios werden allgemein vorhandene Ressourcen genutzt. Hier erfährt das Projekt eine qualitativ hochwertige Umsetzung mit individueller Betreuung“, begründet Jury-Mitglied Dr. Martin Lederle, Stadtlohn bei Münster, die Auszeichnung des Projektes.

\section{DiaKoPf - Diabetologische Kompetenz in Pflegeeinrichtungen}

Ein umfassendes Versorgungskonzept, in dessen Zentrum eine Diabetes-Nurse als Ansprechpartnerin und Multiplikatorin fungiert, führt im Therapie- und Schulungszentrum für Diabetologie der Sana Klinik Oldenburg zu deutlichen Verbesserungen in den Einrichtungen und zu einem sichereren Umgang der Patienten mit der Erkrankung. Die Einbindung aller Beteiligten in die Versorgung älterer Menschen war einer der Gründe, das Projekt auszuzeichnen, erklärt Jury-Mitglied Dr. Dr. Andrej Zeyfang, Stuttgart: „Das sektorenübergreifende Versorgungskonzept, die Beteiligung von Kranken- und Altenpflegekräften, Ärzten und DiabetesberaterInnen mit Bündelung der Kompetenzen zur besseren Versorgung der älteren Patienten halten wir für unterstützungswürdig. Überzeugt hat uns schließlich auch die Nutzung einheitlicher Schulungsprogramme mit etablierten strukturierten Konzepten und die Qualifizierung aller Beteiligten sowie die Zukunftsvision eines dokumentierten Qualitätsmanagements.“ 
Zum Versorgungskonzept gehören Mitarbeiterschulungen in Anlehnung an FoDIAl (Fortbildung Diabetes in der Altenpflege), Fallbesprechungen mit Altenpflegerinnen und Qualitätszirkel der Hausärzte. Schulungen geriatrischer Diabetiker nach SGS (Strukturierte geriatrische Schulung) und Informationsabende für Angehörige und Betroffene ergänzen das Konzept. Der ganzheitliche Ansatz führt nicht nur zu einer besseren Lebensqualität der Bewohner, er führt auch zu mehr Zufriedenheit der Pflegekräfte, berichtet Projektleiter Lars Hecht: „Die Teilnehmer von DiaKoPf fühlen sich sicherer im Umgang mit Diabetes. Die Hausärzte sind sehr zufrieden mit DiaKoPf, da sie in den Einrichtungen nun Ansprechpartner haben, die zu Diabetes kompetent Auskunft geben können.“

Die Zukunft wird älter. Zeit zu handeln Der SilverStar Förderpreis ist das Herzstück der neuen Initiative der BerlinChemie AG „Die Zukunft wird älter. Zeit zu handeln.“, die Torsten Flöttmann, Berlin-Chemie AG, während der Preisverleihung vorstellte. „Die Menschen werden älter. Schätzungen zufolge werden im Jahr 2030 bereits über $27 \%$ aller Deutschen älter als 65 Jahre sein. Das bedeutet aber auch, dass es immer mehr Menschen mit Alterserkrankungen geben wird. Die Zukunft stellt also große Herausforderungen an die ganze Gesellschaft, die wir als Unternehmen annehmen“, so Torsten Flöttmann in seinem Vortrag.

Das Engagement für ältere Menschen hat bei der Berlin-Chemie AG Tradition. Zahlreiche Projekte beschäftigen sich bereits mit dem Thema Altersmedizin, und auch in Zukunft möchte das Unternehmen sich zunehmend für ein gesundes Älterwerden einsetzen. „Das können wir aber nicht alleine. Wir möchten verstärkt in den Dialog mit allen Beteiligten des Gesundheitswesens treten, um langfristige Konzepte für größtmögliche Lebensqualität und Selbständigkeit bis ins hohe Alter zu entwickeln. Mit der Initiative möchten wir dazu einen aktiven Beitrag leisten."

Alle Informationen zum SilverStar-Förderpreis sind auf www.silverstar-preis.de zu finden. Die Bewerbungsunterlagen für den SilverStar 2012 werden dort zeitnah zur Verfügung stehen.

Quelle: „SilverStar - Aktiv für ältere Patienten“, Preisverleihung und Symposium der BerlinChemie AG im Rahmen der 5. Herbsttagung der Deutschen Diabetes-Gesellschaft (DDG) am 11. November 2011 in Berlin

Strukturiertes Diabetes-Management

\section{Messen - Zusammenhänge verstehen - Therapie anpassen}

Bei der Behandlung von Menschen mit Diabetes mellitus ist die intensivierte Insulintherapie mit selbstständiger Anpassung der Insulindosierungen die Voraussetzung für einen Therapieerfolg. Deshalb gilt die Blutzuckerselbstkontrolle (BZSK) als eine Kernkomponente der effektiven Behandlung von insulinpflichtigem Typ1- oder Typ-2-Diabetes.

Auf der Grundlage von Auswertungen der Blutglukose-Selbstkontrollwerte werden sowohl durch das Behandlungsteam als auch durch den Patienten notwendige Anpassungen vorgenommen. In der Praxis wird zur Stoffwechselsteuerung als Richtwert bevorzugt der $\mathrm{HbA}_{1 \mathrm{c}}$-Wert eingesetzt. Das kann problematisch sein, denn „der $\mathrm{HbA}_{1 \mathrm{c}}$-Wert reflektiert nur die mittlere Blutglukosekonzentration, Schwankungen werden nicht erfasst, eine frühe und aktive Stoffwechselkontrolle ist nicht möglich“ warnte Prof. Stephan Jacob, Villingen-Schwenningen.

Strukturierte Selbstkontrolle

ist genauer als $\mathrm{HbA}_{1 c}$-Wert

Eine pathophysiologisch sinnvolle und spezifisch auf das BZ-Profil des Patienten ausgerichtete Therapie ist nach Ansicht Jacobs hingegen mit der strukturierten Blutzuckerselbstkontrolle (Pattern Analyse) möglich. Mit der Pattern Analyse werden Blutzuckerverläufe bei Menschen mit Diabetes grafisch und tabellarisch dargestellt und können vom geschulten Diabetesteam auf einen Blick erfasst werden. Schwankungen und Ursachen können so identifiziert werden und die Therapie entsprechend angepasst werden, lange bevor dies im $\mathrm{HbA}_{1 \mathrm{c}}$-Wert erkennbar wird. Auch für den Patienten wird anhand von Mustern die Erkennung von Zusammenhängen plausibel. Dabei können einfach zu handhabende Protokollbögen bis hin $\mathrm{zu}$ hochentwickelten Diabetesmanagement-Instrumenten (Accu-Chek ${ }^{\circledR}$ Smart Pix, Accu-Chek ${ }^{\circledR} 360^{\circ}$ Diabetes Management Software) genutzt werden, die Dr. Ralph Ziegler, Münster, vorstellte. Dabei gilt es, strukturiert in 4 Schritten vorzugehen, wie der Diabetologe erläuterte. Zunächst ist es wichtig, zu erkennen, welche Blutzuckerschwankungen auftreten: Sind es eher
Hypo- oder Hyperglykämien und treten letztere nüchtern oder postprandial auf? Im nächsten Schritt ist es sinnvoll zu überprüfen, ob die Schwankungen zu bestimmten Tageszeiten auftreten und anschließend, ob die Blutzuckerverläufe einem erkennbaren Muster folgen. Hat man diese 3 Schritte durchlaufen, kann die Ursachenanalyse schneller und einfacher erfolgen.

\section{Ergebnisse der STeP-Studie zeigen \\ Erfolg des Selbstmanagements}

Dass dieses Vorgehen auch zum Therapieerfolgt führt, konnte mit der „STeP-Studie (Structured Testing Protocol) gezeigt werden [1]. Bei schlecht eingestelltem $\mathrm{HbA}_{1 \mathrm{c}}\left(\mathrm{HbA}_{1 \mathrm{c}} \geq 7,5 \%\right)$ wurden Menschen mit Typ-2-Diabetes, die nicht mit Insulin behandelt waren, mit einer strukturierten BZSK versus konventioneller Diagnostik verglichen. Aufgrund der durch die BZSK gewonnenen Daten wurde die Therapie adjustiert. Mit der strukturierten BZSK erzielten die Patienten eine erheblich bessere Blutzuckerkontrolle $\left(\mathrm{HbA}_{1 \mathrm{c}}{ }^{-}\right.$ Senkung um $0,3 \%[\mathrm{p}<0,005]$ in der intention to treat und sogar $0,5 \%$ in der per protocol Analyse).

Daniel Bomar, Linkenheim-Hochstetten

Literatur

1 Polonsky WH et al. Diab Care 2011; 34: 262-267

Quelle: Symposium „Mehr erkennen mit der Pattern Analyse, therapieren mit der Patch Pumpe: Neue Meilensteine in Diagnostik und Therapie“ im Rahmen der 46. Jahrestagung der Deutschen Diabetes Gesellschaft (DDG) am 1. Juni 2011. Veranstalter: Roche Diagnostics Deutschland $\mathrm{GmbH}$. 


\section{NovoPen Echo ${ }^{\circledR}$ ersetzt künftig NovoPen ${ }^{\circledR}$ Junior}

Novo Nordisk hat im März 2011 den neu entwickelten Insulinpen NovoPen Echo ${ }^{\circledR}$ eingeführt. Das Injektionsgerät wurde speziell für die Bedürfnisse von Kindern konzipiert und hat sich inzwischen fest im Markt etabliert. Das Vorgängermodell, NovoPen ${ }^{\circledR}$ Junior, wurde zum 31. Oktober 2011 vom Markt genommen.

Der wiederbefüllbare NovoPen Echo ${ }^{\circledR}$ wurde für Kinder und Eltern entwickelt, um ihnen ein noch größeres Gefühl an Sicherheit zu geben: So zeigt die Memory-Funktion des Insulinpens sowohl die Dosis als auch die Zeitspanne seit der letzten Insulingabe an. Dadurch wird das Risiko einer versehentlich doppelt injizierten Dosis verringert. Ein weiterer Vorteil besteht darin, dass die Dosis in halben Schritten von 0,5 bis maximal 30 Einheiten eingestellt und ohne Insulinverlust korrigiert werden kann. Ein Klickgeräusch signalisiert das vollständige Herunterdrücken des Dosisknopfes. Im Vergleich zum Vorgängermodell NovoPen ${ }^{\circledR}$ Junior ist bei dem neuen Pen für die Injektion weniger Kraft nötig.

NovoPen Echo ${ }^{\circledR}$ wurde aufgrund seines guten Designs bereits mit dem interna-

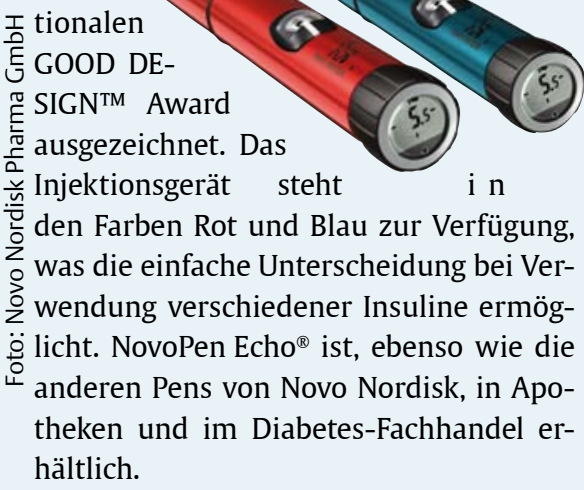

Quelle: 3K Agentur für Kommunikation GmbH, Frankfurt am Main

\section{Euro für das „Life for a Child"“-Programm der IDF erlaufen}

Über 700 Mitarbeiter von Boehringer Ingelheim nahmen am 14. November, dem Weltdiabetestag der International Diabetes Federation (IDF), an einem 3 Kilometer langen Wohltätigkeitslauf teil. Die dabei von den Läufern insgesamt zurückgelegten Kilometer wurden von der Firma in eine finanzielle Unterstützung für das „Life for a Child“-Programm der IDF umgewandelt. Aus dem gleichen Anlass wurden bei der diesjährigen Herbsttagung der Deutschen Diabetes Gesellschaft in Berlin Schrittzähler an Ärzte ausgegeben und die zurückgelegten Schritte ebenfalls in einen finanziellen Beitrag für das „Life for a Child“ Programm umgewandelt. Der so von Boehringer Ingelheim insgesamt für dieses Programm erlaufene Beitrag betrug 20000 Euro.

„Diabetes ist eine globale Epidemie, unter der über 366 Millionen Menschen weltweit leiden'“, so Dr. Birgit Dannhof, internationale Teamleiterin Diabetes bei Boehringer Ingelheim. „Wir freuen uns, dass wir den Weltdiabetestag 2011 und das „Life for a Child“-Programm der International Diabetes Federation unterstützen können. So wie die International Diabetes Federation engagieren auch wir uns langfristig im Bereich Diabetes und setzen uns für ein besseres Verständnis, mehr Aufklärungsarbeit und eine stärkere Prävention ein.“

\footnotetext{
International Diabetes Federation (IDF).
} www.idf.org aufgerufen am 24. Oktober 2011
Mit dem finanziellen Beitrag wird das „Life for a Child“-Programm der IDF gefördert, das innovative und nachhaltige Hilfe für Kinder mit Diabetes in Entwicklungsländern liefert. Zurzeit unterstützt das Programm über 7000 Kinder in 36 Ländern und bietet ihnen medizinische Versorgung sowie das notwendige Wissen zum Thema Diabetes, um mit der Krankheit zu leben ${ }^{1}$. „Unsere Vision ist es, dass kein Kind an Diabetes sterben sollte“, sagte Dr. Graham Ogle, ProgrammManager des „Life for a Child“-Programms der IDF. „In einigen der ärmsten Regionen der Erde sind Todesfälle aufgrund von Diabetes häufig und eine wichtige medizinische Versorgung zum Beispiel durch Insulin oder Blutzucker-Teststreifen nicht gewährleistet. Wir begrüßen daher Initiativen wie diese, damit benachteiligte Kinder weltweit ein längeres und gesünderes Leben führen können.“

Der „Lauf für Diabetes“ von Boehringer Ingelheim war der Höhepunkt einer ganzen Reihe von Aktivitäten im Rahmen der Aktionswoche, die anlässlich des Weltdiabetestags zur Steigerung der Wahrnehmung von Diabetes organisiert wurde. Nicht nur am Hauptsitz von Ingelheim in Deutschland engagiert man sich tatkräftig im Bereich Diabetes - auch in den Niederlassungen weltweit wird der Kampf gegen die Krankheit unterstützt, weshalb eine Vielzahl von Gebäuden des Unternehmens als sichtbare Solidaritätsbekundung blau angestrahlt wurde. Um

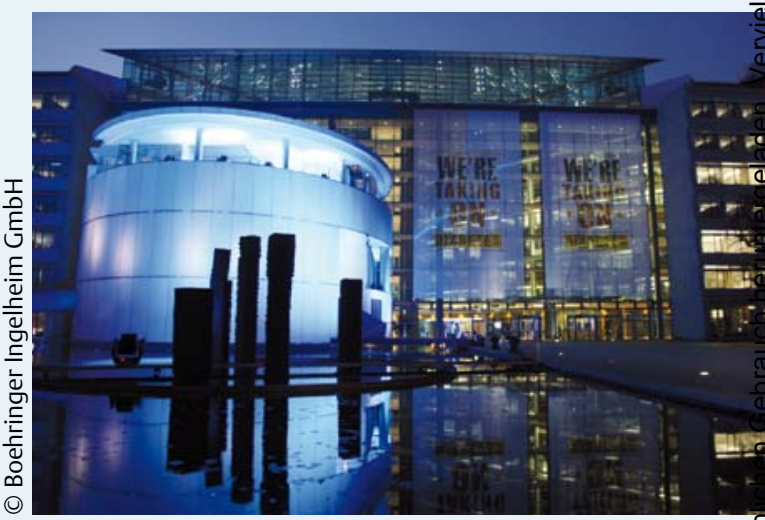

Das Headquarter von Boehringer Ingelheim in Ingelheim am Rhein wurde anlässlich des Weltdiabetestags blau erleuchtet.

die Bedeutung einer gesunden Ernährung und einer aktiven Lebensweise bei der Vermeidung von Typ-2-Diabetes bzw. im Umgang mit der Krankheit zu unterstreichen, standen in der Aktionswoche aber auch eine Vielzahl weiterer Aktivitäten auf dem Programm.

Der Weltdiabetestag wurde 1991 durch das IDF sowie die Weltgesundheitsorganisation WHO ins Leben gerufen. Ziel war und ist es, über die gesundheitlichen Folgen der weltweit über die gesundheitlichen Folgen dieser weltweit steigenden Prävalenz der Erkrankung zu informieren. Im Fokus diesen Jahres stehen zum dritten Mal im Zuge einer fünfjährigen Kampagne „Diabetes Aufklärung und Vorsorge“.

Quelle: PM Boehringer Ingelheim GmbH 